\title{
A Pilot Study to Understand the Acceptance of Clinical Research Industry in Application of Risk-Based Quality Management System to Clinical Trials
}

\author{
Heema Desai ${ }^{1 *}$, Kaushal Kapadia ${ }^{2}$ \\ 'Clinical Research, M.Sc., Texila American University. \\ ${ }^{2}$ Clinical Research, Ph.D., Clinical Research Consultant, India.
}

*Address for Correspondence: Heema Desai, Clinical Research, M.Sc., Texila American University; E mail: researchcrest@yahoo.com

Received: 11 September 2020; Accepted: 26 October 2020; Published: 31 October 2020

Citation of this article: Desai H, Kaushal K (2020) A Pilot Study to Understand the Acceptance of Clinical Research Industry in Application of Risk-Based Quality Management System to Clinical Trials. Rea Int J of Community med and Pub Health. 1(1): 037-041. DOI: $10.37179 /$ rijcmph.000007.

Copyright: (C) 2020 Desai $\mathrm{H}$, et al. This is an open access article distributed under the Creative Commons Attribution License, which permits unrestricted use, distribution, and reproduction in any medium, provided the original work is properly cited.

\begin{abstract}
Aim: To understand the acceptance of clinical research industry in application of risk-based quality management system to clinical trials.

Methodology: A survey-based study was conducted by taking feedback from 30 clinical research professionals with the help of validated questionnaire comprising of 10 questions. As it was a pilot study the analysis was a considerably basic one by using a percentage method.

Results: $80 \%$ (24 participants) feel that failure to identify priorities, poor risk identification and risk mitigation is the major quality issue, $86.67 \%$ (26 participants) mentions that regulatory authorities focuses on training and quality assurance and auditing for developing quality systems, 90\% (27 participants) opine that re-defining key risk and performance indicator's is the most important parameter for RBQM implementation. There is $100 \%$ acceptance for the basic idea of risk-based quality management is the identification of the risks on a continuous basis for risk-bearing activities, that Risk based quality management involves Risk Assessment, Risk control, Risk review, Risk management, Risk Communication and agree that while implementing the quality management, the prime objective is to provide assurance that the rights, safety and well-being of trial subjects are protected.

Conclusion: Acceptance of RBQM by the industry is in lines with the guidelines and systems. Collaboration with CROs (i.e. outsourcing model) will always continue to be the best model in bringing the better quality in timely manner.
\end{abstract}

Keywords: Risk-Based Monitoring, Quality Management, RBQM, Clinical Trials, RBM, Key aspects of RBM, Monitoring.

\section{Introduction}

Quality in context with clinical research is commonly defined as fitness for purpose. Clinical research is about generating information to support decision making while protecting the safety and rights of participating subjects. The quality of information generated should therefore be enough to support good decision making. ICH GCP E6 R2; section 5.1 speaks of quality assurance and quality control
Quality Assurance: All those planned and systematic actions that are established to ensure that the trial is performed and the data are generated, documented (recorded), and reported in compliance with Good Clinical Practice (GCP) and the applicable regulatory requirement(s) [1].

ISO 9000 is defined as a set of international standards on quality management and quality assurance developed to help companies effectively document the quality system elements needed to maintain 
an efficient quality system. They are not specific to any one industry and can be applied to organizations of any size. Section 5.1 of ICH GCP and Article 2 of the GCP Directive 2005/28/EC states that the sponsor or Contract Research Organizations (CRO) needs to implement and maintain the systems for quality assurance and quality control and secure the quality of every aspect of the trial. These quality management procedures aim at providing assurance that the rights, safety, and well-being of trial subjects are protected, and that the results of the clinical trials are credible. The key elements of the quality system include: development of documented procedures and validated methods, maintaining of audit trails, training of sponsor/ CRO personnel, validation of computerized system which includes quality control i.e. use of centralized monitoring systems and quality assurance which includes external and internal audits [2].

The fundamental objective of risk-based quality management is the identification of the risks on a continuous basis i.e. end-to-end trial execution from the design, conduct, evaluation, and reporting of clinical trials. The quality management system needs to use a risk-based approach: Risk Identification, Risk Control, Risk Review, Risk communication and Risk Reporting. All quality management processes are dynamic. Thus, continuous improvement is only ensured, when quality management processes are constantly adapted by collecting and using information on on-going basis [2]. Riskbased quality management approach includes the following which is currently practiced by the industry to achieve the best quality systems.

\section{Risk Assessment}

Assessing risk involves two steps risk identification and risk evaluation. For system level risk identification, the systems should be analyzed to identify potential risk that could affect organization and for project level risk identification, trial specific information should be analyzed to identify the risks. Risk evaluation includes establishment of priorities at the time of study design throughout the different stages of the trial. The data collection and monitoring tools should reflect the priorities [2].

\section{Risk Control}

The purpose of Risk control is to reduce the risk to an acceptable level. During risk control, a mitigation plan should be prepared and implemented. The amount of effort used for risk control should be proportional to the significance of the risk and the importance of the process or outcome exposed to identified risk [2].

\section{Risk Mitigation}

Risk mitigation involves actions taken to reduce the unacceptable risk. Unacceptable risk can be defined as the risk that probes considerable impact on subject's safety and rights including the credibility of data. Risk mitigation plan should be project specific and can include protocol design, designing of monitoring plan, audit, and data management plans to identify priorities and risk. Risk mitigation can also include process for identification and escalation of risk.

\section{Risk Review}

Risk review mandates the integration of risk assessment and risk control with risk management tools and the communication of the results and data associated to the risk identified and the documentation of the actions needed to mitigate the risk [2].

\section{Reporting Quality}

The feedback from the risk review should be analyzed and summarized. The analysis report will include variable measurement, their timing, assessment of deviation and missing data. Additional information can be achieved by well-designed intra and inter site variance analysis on single or multiple variables. Trend Analysis should be done in relation to the overall impact on the scientific benefits and usability of the generated data as established through priority setting and identification of risks and can be supplemented with information on process compliance based on monitoring/ data management reports [2]. Implementing Risk-Based Quality Management becomes one of the crucial part of the process and hence it is important for us to understand what are the features enlisted under it and how it is practiced.

\section{Redefining the sponsor/CRO relationship}

As per ICH GCP R2 sponsors/CROs are responsible for managing risk in trial. To achieve compliance, they should redefine a communication plan for sharing trial updates, response strategies and systems that will be used to share data and monitoring outcomes. The challenges in budgeting and resource allocation can be overcome by considering pay-for-performance criteria into the project plan [3].

\section{Redefining key risk and performance indicators}

The RBQM platform allows the CRO and sponsor to define KRIs and KPIs and create weighted scorecards to more efficiently track outliers and risk signals based on phase of research, biomarkers, protocol [3]. KRIs can be defined as a measure that indicates how risky an activity or process can be. KRIs can be identified during risk assessment phase of clinical trial.

\section{Examples of KRIs that may be important are:}

1. Safety: KRI related to potential AE

2. Investigational Product: KRI related to issues arising of IP accountability, dosage, and administration

3. Recruitment: Monitoring of safety and efficiency of subjects.

4. Issue Management: KRI dealing with general issues.

5. Data Quality: This KRI tackles issues arising out of abnormal trends.

6. Resourcing and maintenance: KRIs dealing with staff training and storage and calibration of equipment's.

\section{Choosing the right technology}

A right technology system should be selected by sponsor/ CROs which could integrate and aggregate data in real time. An effective RBQM solution should provide the necessary integration, transparency, and analytics capabilities to drive real-time risk identification and mitigation [3].

\section{Retraining Staff}

Site Monitors and site staff will need training on how to operate in an RBQM environment, including how risk indicators are defined and configured and the expected mitigation responses when risks occur. Data analytics will play an important role in supporting these programs [4]. 
Citation: Desai H, Kaushal K (2020) A Pilot Study to Understand the Acceptance of Clinical Research Industry in Application of Risk-Based Quality Management System to Clinical Trials. Rea Int J of Community med and Pub Health. 1(1): 037-041. DOI: $10.37179 /$ rijcmph.00007.

\section{How is the Industry Responding?}

Even though clinical research industry has not yet achieved $100 \%$ adoption to RBQM; still large organization are well on the path to implementation. Many of those organizations seem to have mature processes to implement risk planning and monitoring. Small and midsize organizations are primarily in the early stages of implementation. Those organizations are beginning to accept the requirement to transform their operational processes and methodology [5]

\section{Methodology}

Qualitative methodology was used to explore and to understand how clinical research industry is implementing risk-based quality management and the perceived issues and possible systems that will help easy implementation. It was done by conducting a survey for which the survey questionnaire was prepared, and questions were validated before circulation within clinical research professional across the country. We received perspectives from clinical research professional having more than 6 years of experience in the industry. Thirty professionals participated in survey, and all had direct or indirect experience of working with risk-based monitoring model. In this study, a questionnaire- based survey, the prepared questionnaire was distributed to industry professional via an email/text message (link for google forms). This message was sent to participants and the expected number of survey response was collected in a duration of 4 months.

\section{Flowchart:}

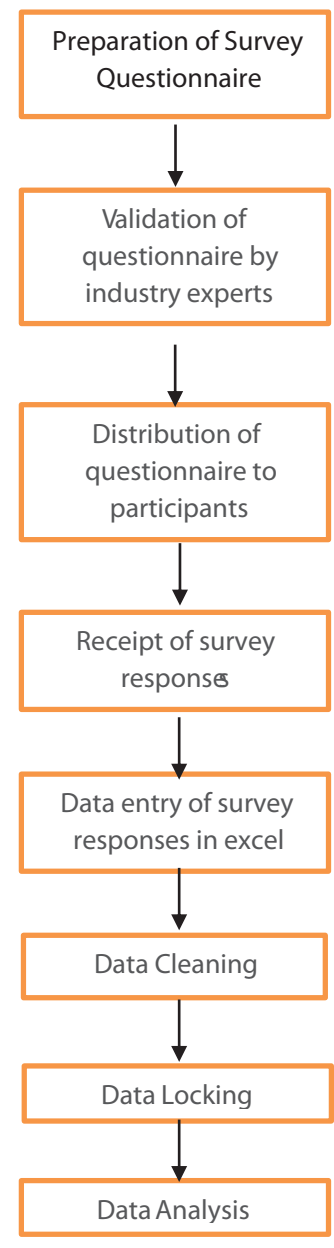

\section{Results \& Discussion}

The survey questionnaire (attached as Appendix I) designed for the study was having 10 closed ended questions. Key results of these are explained below. Questionnaire was designed based on the reviewed literature and the same was validated with the help of an industry expert. Level 1 analysis was conducted as this is a pilot study which was conducted with a small sample size. Based on the survey data, $100 \%$ of participants agreed that RBQM is the identification of the risks on a continuous basis for risk-bearing activities throughout the course (end-to-end) of clinical trials., $96.67 \%$ believed that RBQM application will facilitate better and informed decision making and better utilization of available resources and $63.33 \%$ agreed that clinical research industry is prepared to shift from traditional monitoring model to RBQM. Knowing the General Quality issues in Clinical research was equally important as this would lead to identification of the right corrective and preventive actions.

It was observed that, $80 \%$ (24 participants) feel that failure to identify priorities, poor risk identification and risk mitigation is the major quality issue, $53.33 \%$ (16 participants) feel lack of proportionality, knowledge and capabilities is the second major quality issue, 40\% (12 participants) believe cost of development and development deadlines is the quality issue followed by $20 \%$ (6 participants) who feel regulatory environment is the issue and $10 \%$ (3 participants) indicated that Lack of time to do proper study training; huge workload is the quality issue. At the end of the day we all look towards getting the best quality and for that with the guidelines and other ethical norms we also need to know as to what the Expectations of Regulatory Authorities are while developing quality systems.

(Figure 2) survey responses indicate that $86.67 \%$ (26 participants) regulatory authorities focuses on training and quality assurance and auditing, $73.33 \%$ (22 participants) feel that document management, record retention and reporting is the second quality expectation of the regulatory authority, 66.67\% (20 participants), 56.67\% (17 participants) and $46.67 \%$ (14 participants) feel that CAPA, Policies and procedures and personnel roles and responsibilities respectively are the regulatory authorities expectation followed by $6.67 \%(2$ participants) who feel Inspection readiness and ethical practices, Regular Oversight of study are also the regulatory expectation. In continuation we also need to know, how much is the industry prepared to accept the shift from on-site monitoring (the traditional model) to RBQM:

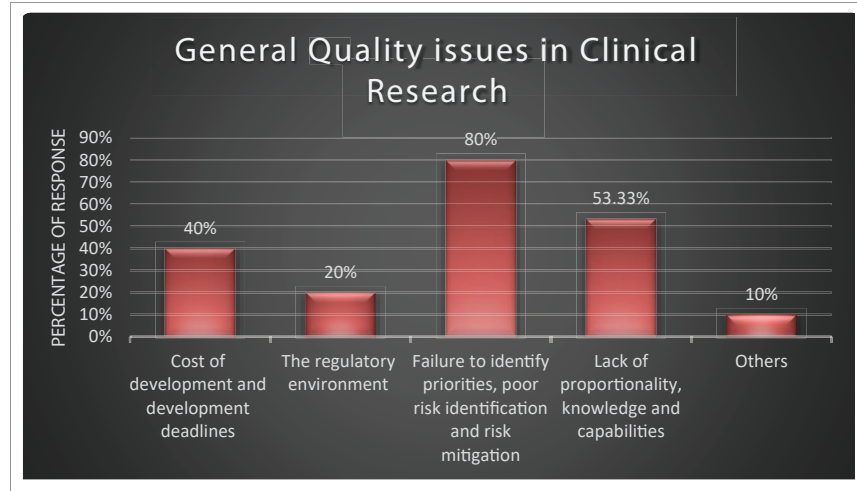

Figure 1: General Quality Issues in Clinical Research. 
Citation: Desai H, Kaushal K (2020) A Pilot Study to Understand the Acceptance of Clinical Research Industry in Application of Risk-Based Quality Management System to Clinical Trials. Rea Int J of Community med and Pub Health. 1(1): 037-041.

DOI: $10.37179 /$ rijcmph.00007.

(Figure 3) is indicative that $63.33 \%$ (19 participants) feel that industry is ready to accept the shift from on-site monitoring to RBQM whereas $36.67 \%$ (11 participants) feel's that industry is still not ready. Which we would still expect to improve if there is some support, so the next question. Would Collaboration with CROs will make sponsor accept RBQM efficiently (Figure 4) indicates that 83.33\% (v25 participants) accepts that collaboration with CROs will make RBQM acceptance easy and fast for sponsors while $16.67 \%$ (5 participants) opine the otherwise. And this what the industry is working towards to, "the outsourcing model" A sub-group analysis was conducted to understand the comparison between industry preparedness for shift to RBQM v/s parameters needed for RBQM implementation, as this will help us in knowing which parameters can help towards understanding the parameters which would be needed in implementation towards increasing the industry preparedness.

(Figure 5) is a comparison between industry preparedness to shift from on-site monitoring to RBQM v/s parameters for RBQM implementation. There is difference of $1.92 \%$ (4 participants) when vendor selection is considered followed by $6.22 \%$ (7 participants) difference observed for sponsor/CRO to re-define interaction to share trial updates and response strategies. Next is $12.92 \%$ ( 9 participants) difference observed in re-defining key risk and performance indicator's followed up $25.83 \%$ (10 participants) difference for integrating change management strategy, 27.75\% (2 participants) difference was observed for correct technology selection parameter and $41.15 \%$ (10 participants) difference was seen in parameter related to re-training of staff. Another sub-group analysis was done

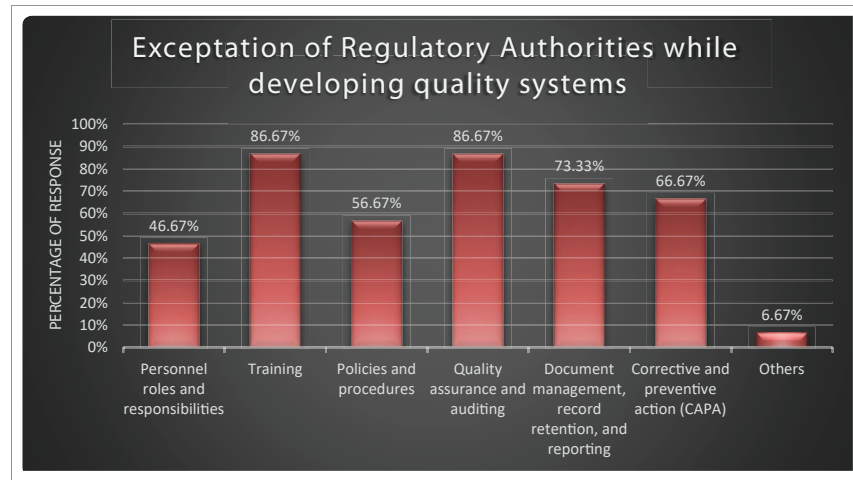

Figure 2: Expectation of Regulatory Authorities while developing quality systems.

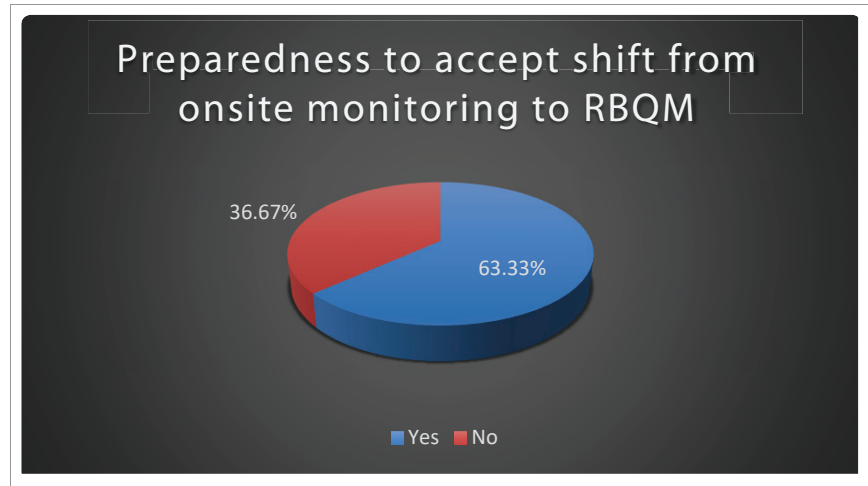

Figure 3: Industry preparedness to accept the shift from on-site monitoring to RBQM

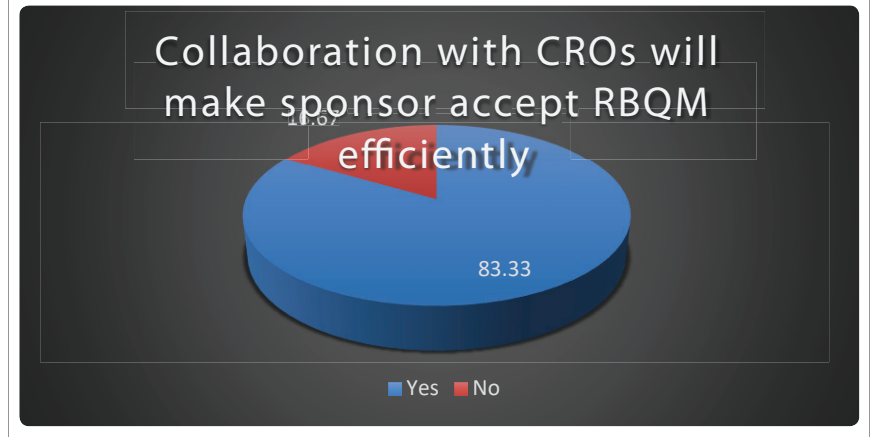

Figure 4: CRO collaboration will make sponsor accept RBQM efficiently.

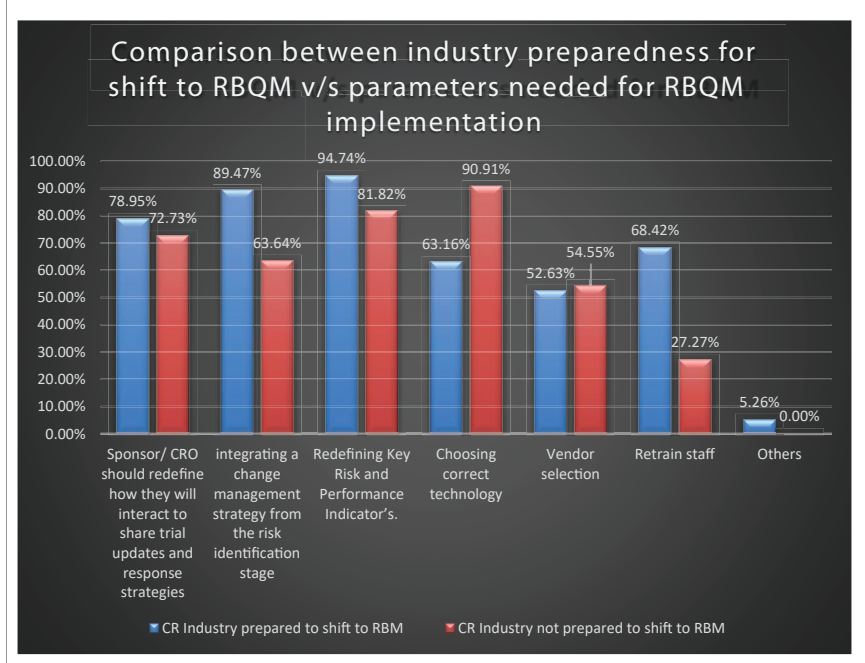

Figure 5: Industry preparedness for shift to RBQM v/s parameters needed for RBQM implementation.

to understand if there is poor RBQM acceptance by sponsors and if there is a CRO collaboration would this combination make RBQM acceptance easy and faster (Figure 6) illustrates that 80.95\% (21 participants) feel that even after seven years of RBQM introduction sponsor is still have poor acceptance of RBQM and collaboration with CROs will help sponsors accept RBQM more easily and efficiently [6] .

\section{Conclusion}

The present study indicates the acceptability of RBQM in clinical research industry. The study additionally addresses the general quality issues in clinical research and industry preparedness in shifting from traditional mind-set. The prime motive of risk-based quality management is the identification of the risks on a continuous basis throughout the course (end-to-end) of clinical trials and the study results are indicative that industry looks forward in executing the RBQM to make informed decisions by identifying and mitigate the risk at an early stage of the trial. The study unanimously indicates that any trial conducted should provide assurance that the rights, safety and well-being of the trial subjects are protected, and that the results of the clinical trials are credible; to achieve this defining key risk and performance indicator and integrating change management strategy at the very beginning of the trial will prove to be beneficial.

Although, the sponsors are positively adapting the RBQM allying (C) 2020 MSD Publications. All rights reserved. 
Citation: Desai H, Kaushal K (2020) A Pilot Study to Understand the Acceptance of Clinical Research Industry in Application of Risk-Based Quality Management System to Clinical Trials. Rea Int J of Community med and Pub Health. 1(1): 037-041.

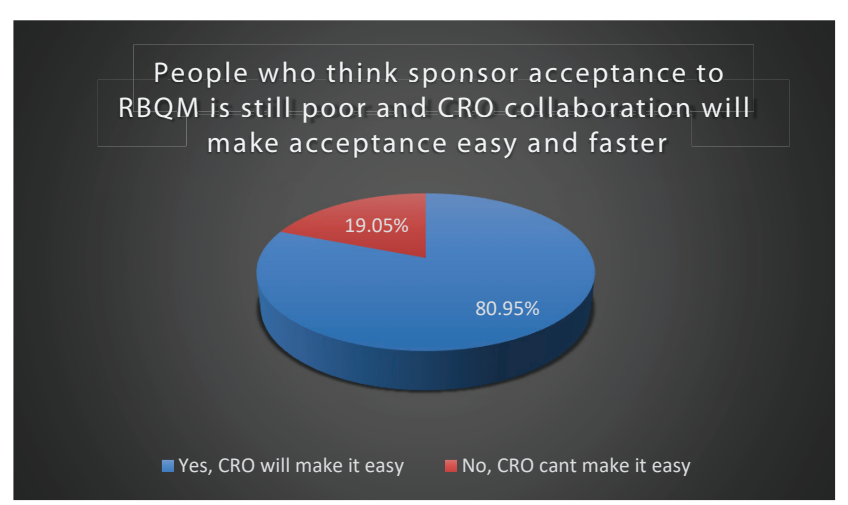

Figure 6: People who think sponsor acceptance to RBQM is still poor and $\mathrm{CRO}$ collaboration will make acceptance easy and faster.

with CRO will ease the process of adaption and implementation of RBQM in the clinical trials. Furthermore, the quality systems should be developed in a manner that facilitates quality assurance and auditing process.

\section{References}

1. (2020) E6 (R2) Good Clinical Practice: Integrated Addendum to ICH E6 (R1). Link: https://bit.ly/2HAyusq

2. (2020) Reflection paper on risk-based quality management in clinical trials EMA/269011/2013. Link: https://bit.ly/2TurDDA

3. Regan B (2018) Moving Beyond Risk-based Monitoring: Improving Quality and Performance with Real-time Management; Pharma Voice: 41. Link: https://bit.ly/31PFQzt

4. WHAT IS THE ISO 9000 STANDARDS SERIES? Link: https://bit. ly/31PcW2p

5. Abraham A (2019) New Survey Measures the Pulse of RBM \& ICH-E6 (R2) Adoption in the Clinical Trials Industry. Link: https://bit. ly/3jvRc1z

6. Desai H, Kapadia K (2020) A pilot study to understand the challenges and benefits of implementing Risk based monitoring in clinical research, MAR Pharmaceutical Sciences. Link: https://bit. Iy/35KVTjs 